\title{
ON THE SUFFICIENT CONDITIONS IN THE CALCULUS OF VARIATIONS.
}

BY DR. E. R. HEDRICK.

(Read before the American Mathematical Society, December 28, 1901.)

The sufficient conditions in the calculus of variations have recently received a great deal of attention; ${ }^{*}$ and it would seem fitting that attempts be made to simplify their discussion whenever possible, and to render the agreement more exact between the known necessary and the known sufficient conditions. Such is the purpose of this paper, which also seeks to present the sufficient conditions in compact form. The work will to a large extent follow lectures delivered at Göttingen by Professor Hilbert, 1899-1901.

\section{Hilbert's Invariant Integral. $\dagger$ Weierstrass's Sufficient Condition.}

Let us consider a simple definite line integral

where

$$
I=\int_{x_{0}}^{x_{1}} f\left(x, y, y^{\prime}\right) d x
$$

$$
y^{\prime}=\frac{d y(x)}{d x}
$$

and where $f$ is an analytic function of the three arguments $x, y, y^{\prime}$, in a certain region $R$. Let us then restrict ourselves to the consideration of curves of integration contained in a realm $B$, consisting of curves of the type

$$
y=\phi(x)
$$

* Du Bois-Reymond, Mnth. Annalen, 15 ; Kneser, Lehrbuch der Variationsrechnung, and many memoirs in Math. Annalen; Osgood, Annals of Math., $2 \mathrm{~d}$ ser., vol. 2, no. 3, and Transactions Amer. Math. Soc., vol. 2, pp. 166, 273 ; Whittemore, Annals of Math., 2d ser., vol. 2, no. 3; Bolza, Ithaca Colloquium (summer meeting, Amer. Math. Soc., Aug., 1901), unpublished, and Transactions Amer. Math. Soc., vol. 2, p. 422; Weierstrass, lectures at Berlin, 1879-1882, unpublished ; Hilbert, lectures at Göttingen, 1899-1901, unpublished, etc.

† Compare Osgood, Annals of Math., l. c., where Hilbert's proof is given. 
where $\phi(x)$ together with its first derivative is a single valued and continuous function of $x$ in the interval $x_{0} \leqq x \leqq x_{1}$, and where $\phi\left(x_{0}\right)$ and $\phi\left(x_{1}\right)$ have certain fixed values $y_{0}$ and $y_{1}$ respectively. If the value of the integral $I$ taken along a curve

$$
y=y(x)
$$

of the realm $B$ be less than the value of $I$ taken along any other curve of the realm $B$,

$$
y=Y(x) \equiv y(x)+\eta(x)
$$

where $Y(x)$ is restricted by the same conditions as $y$ and also by

$$
|\eta(x)|<\delta, \quad\left|\eta^{\prime}(x)\right|<\delta, \quad x_{0} \leqq x \leqq x_{1},
$$

$\delta$ being a positive constant chosen at pleasure, then the curve (3) is said to render the integral $I$ a weak minimum, as compared to curves of the realm $B$. If in place of the conditions (5) we merely require that

$$
|\eta(x)|<\delta, \quad x_{0} \leqq x \leqq x_{1},
$$

then the curve (3) is said to render $I$ a strong minimum.* If in addition to the condition (5) $[$ or (6)] we require that

$$
\begin{gathered}
\eta\left(x_{i}\right)=0 ; \quad x_{0}+(i-2) \cdot \delta \leqq x_{i} \leqq x_{0}+(i-1) \delta \leqq x_{1} ; \\
\left(i=2,3, \cdots n,(n+1) ; n \cdot \delta=x_{1}-x_{0}\right),
\end{gathered}
$$

then we will say that the curve (3) renders $I$ a limited weak [or strong] minimum. $\dagger$

It can be shown $\ddagger$ that if the curve $(3)$ is to render $I$ a minimum (of any kind), $y(x)$ must satisfy Lagrange's equation

$$
\frac{d}{d x}\left(\frac{\partial f}{\partial y^{\prime}}\right)-\frac{\partial f}{\partial y}=0
$$

The solutions of this equation are called extremals.

Let us now assume: $\S$

* For the above, of. Osgood, Annals of Math., l. c., p. 106.

†This is what Hilbert calls "ein Minimum bei stückweiser Variation."

† See Whittemore, $l$. $c$., p. 130, where Hilbert's proof is reproduced.

SCompare Osgood, Annals of Math., l. c., p. 113 ; Transactions Amer. Math. Soc., l. c., p. 168. 
(a) That an extremal $C$,

$$
y=y(x)
$$

can be obtained, such that $y_{0}=y\left(x_{0}\right)$ and $y_{1}=y\left(x_{1}\right), i$. e., such that $C$ passes through the two fixed points $P:\left(x_{0}, y_{0}\right)$ and $Q$ : $\left(x_{1}, y_{1}\right)$ given in the problem.

(b) That a one parameter family of extremals

$$
y=\phi(x, \alpha)
$$

can be obtained, where $\phi$ is an analytic* function of $x$ and $\alpha$, $x_{0} \leqq x \leqq x_{1},|\alpha|<\epsilon$; and where

(c) That

$$
\phi(x, 0) \equiv y(x) .
$$

$$
\frac{\partial \phi(x, 0)}{\partial \alpha} \neq 0, \quad x_{0}<x \leqq x_{1} .
$$

It then follows that through any point of a suitably chosen neighborhood $N$ about the curve $C$, one and only one extremal of the family (10) can be passed; and that (10) can be solved for $\alpha$ in terms of $x$ and $y$,

$$
\alpha=\psi(x, y),
$$

where $\psi(x, y)$ is a singly valued analytic function of $x$ and $y$, in the neighborhood $N$. Hence also

$$
y^{\prime}=\frac{\partial \phi(x, \alpha)}{\partial x}
$$

can be expressed as a singly valued analytic function of $x$ and $y$,

in the neighborhood $N$.

$$
y^{\prime}=p(x, y)
$$

Hilbert now considers the integral

$$
J=\int_{x_{0}}^{x_{1}}\left[f(x, y, p)+\left(y^{\prime}-p\right) f_{p}(x, y, p)\right] d x
$$

taken along any curve $\bar{C}, y=Y(x)$, joining $P$ and $Q$, and

* Since $f$ has been assumed analytic in $x, y, y^{\prime}$, it follows that the coefficients in (8) are analytic, and hence that the solutions of (8) are analytic. 
lying in the neighborhood $N$, where $y$ and $y^{\prime}$ refer to the curve of integration and $p$ denotes the above function $p(x, y)$, and where $f_{p}$ means $\partial f / \partial p$. It is then shown that this integral $J$ is independent of the path of integration,* $i$. e., independent of $Y(x)$. It is seen at once that $J_{C}=I_{C}$, where $J_{C}$ denotes the value of the integral $J$ taken along the curve $C$, and so on. But $J_{\bar{C}}=J_{C}$ since $J$ is independent of the path. Hence the necessary and sufficient condition for a minimum, which is that $I_{\bar{C}}>I_{C}$, becomes at once $I_{\bar{C}}>J_{\bar{c}}$, or $(I-J)_{\bar{c}}>0$, or

$$
\left.\int_{x_{0}}^{x_{1}} E\left(x, y, y^{\prime}, p\right) d x\right]_{\bar{c}}>0 \text {, }
$$

where $\bar{C}$ is any curve in $N$ other than $C$, and where

$$
E\left(x, y, y^{\prime}, p\right) \equiv f\left(x, y, y^{\prime}\right)-f(x, y, p)-\left(y^{\prime}-p\right) f_{p}(x, y, p) .
$$

It follows that

$$
E\left(x, y, y^{\prime}, p\right) \geqq 0, \quad x_{0} \leqq x \leqq x_{1}
$$

for all $x, y$ near the curve $C$, for the function $p$ found above, and for any $y^{\prime}$ whatever, is a sufficient condition for a strong minimum, $\dagger$ where the sign of equality is to hold only along the extremals (10). And, moreover, the condition

$$
E\left(x, y, y^{\prime}, p\right) \geqq 0, \quad x_{0}<x<x_{1},
$$

for all $x, y, p$ on $C$ and for all $y^{\prime}$ whatever, is a necessary condition for a strong minimum. $\dagger$ For if, for simplicity, the family of extremals (10) be so taken as all to pass through $P:\left(x_{0}, y_{0}\right)$, then a curve can be found which renders the integral less than $C$ does, in case $E<0$ at any point of $C$ in any direction $y^{\prime} \neq p$. For we need only take as the comparison curve desired an extremal other than $C$, together with a curve which cuts $C$ at this point in the given direction. The integral $\int E d x$ taken along such a curve is certainly negative.

Let us now consider a fixed point $R:(x, y)$, on the curve $C$, and $f\left(x, y, y^{\prime}\right)$ as a function of $y^{\prime}$ alone, for these fixed values of $x$ and $y$. Let us then draw in a $y^{\prime} f$-plane the curve

* See Osgood, Annals of Math., l. c., p. 122.

$\dagger$ Provided, of course, that conditions $(a),(b),(c)$ hold. 
$f=f\left(y^{\prime}\right) . \quad$ By $(16)$ the inequality $E>0$ becomes

$$
\frac{f\left(y^{\prime}\right)-f(p)}{y^{\prime}-p} \lessgtr f_{p}(p) \text { according as } y^{\prime}-p \lessgtr 0 .
$$

Let $\theta$ be the angle made by the axis of $y^{\prime}$ with the tangent to the $y^{\prime} f$-curve at the fixed point where $y^{\prime}=p$. And let $\psi$ be the angle between the $y^{\prime}$ axis and the chord joining this fixed point to a variable point where $y^{\prime}=y^{\prime}$. Then, as is easily seen from a figure,

$$
\tan \theta=f_{p}(p) \text { and } \tan \psi=\frac{f\left(y^{\prime}\right)-f(p)}{y^{\prime}-p} .
$$

Hence the inequality (19) becomes

$$
\tan \psi \lessgtr \tan \theta \quad \text { according as } y^{\prime} \lessgtr p .
$$

But this is surely fulfilled (except, of course, for $y^{\prime}=p$ ) provided the $y^{\prime} f$-curve is always concave upward, the $f$ axis having been taken vertical, for the range of values of $y^{\prime}$ considered.

Hence the condition

$$
\frac{\partial^{2} f}{\partial y^{\prime 2}}>0
$$

for all $x, y$ on the curve $C$ and for all $y^{\prime}$ considered, is a suffcient condition for a minimum.* For if (21) is satisfied, the $y^{\prime} f$-curve for every point $(x, y)$, on or near $\dagger C$, will be concave upwards, for the whole range of values of $y^{\prime}$ considered, and hence, by the above, the Weierstrass sufficient condition (17) will be satisfied. $\ddagger$

The condition (21) is, however, by no means necessary. A necessary condition usually attributed to Legendre resembles it in form. This necessary condition requires that $\frac{\partial^{2} f}{\partial{y^{\prime}}^{\prime}} \geqq 0$ for all

* Weak or strong, according as the values of $y^{\prime}$ are restricted, or not. Here, as elsewhere, the region $R$ of page 11 must not be overstepped. In particular, the value $y^{\prime}=\infty$ must always be carefully investigated.

† On account of the continuity of $\frac{\partial^{2} f}{\partial y^{\prime 2}}$ in $x, y, y^{\prime}$.

$\ddagger$ This also follows from $\left.\operatorname{Limit}_{y^{\prime}=p}\left[\frac{E\left(x, y, y^{\prime}, p\right)}{\left(y^{\prime}-p\right)^{2}}\right]=\frac{\partial^{2} f}{\partial y^{\prime 2}}\right]_{y^{\prime}=p}$. 
$x, y$ on the curve $C$ and for $y^{\prime}=p .^{*} \quad$ A proof of this theorem is given in the following section.

\section{Limited Variation. Legendre's Condition.}

If we now consider limited variation only, we have the following theorem: Lagrange's, Legendre's narrower, $\dagger$ and Weierstrass's $\ddagger$ conditions are together sufficient for a limited strong minimum.

We suppose first found a solution $C$ of Lagrange's equation connecting the points $P$ and $Q$. Around any point of $C$ a region can so be bounded off that one and only one extremal exists joining the given point to any point of the region; which follows at once from the Cauchy-Kowalewski existence theorems, since $\left.\frac{\partial^{2} f}{\partial y^{\prime 2}}\right]_{C} \neq 0$ at such a point, from the narrower condition of Legendre. If now we require that $\eta(x)=0$ at least once in the interior of each of a set of regions such that the CauchyKowalewski solutions starting from the one end point are unique up to the other end point, we surely have in each such region a satisfactory field for the Weierstrass-Hilbert theory. It follows that the Weierstrass-Hilbert reasoning can be applied, and hence, if the Weierstrass condition is satisfied, the curve $C$ actually renders the integral $I$ a limited strong minimum.

Likewise it is clear that Lagrange's, and Legendre's narrower conditions alone are together sufficient for a limited weak minimum. For Legendre's condition, $\left.\frac{\partial^{2} f}{\partial y^{\prime 2}}\right]_{C}>0$ is satisfied. But this insures $\S$ the fulfillment of Weierstrass's sufficient condition, by the end of Section 1 , since $\frac{\partial^{2} f}{\partial y^{\prime 2}}$ is a continuous func-

* For this condition we shall use the notation $\left.\frac{\partial^{2} f}{\partial y^{\prime 2}}\right]_{C} \geqq 0$; and we shall call it, temporarily, Legendre's broader condition ; later, Legendre's necessary condition. The corresponding condition, $\left.\frac{\partial^{2} f}{\partial y^{\prime 2}}\right]_{C}>0$, where the sign of equality may not hold, we shall call, temporarily, Legendre's narrower condition ; later, sufficient. It is readily seen that $\left.\frac{\partial^{2} f}{\partial y^{\prime 2}}\right]_{C}>0$ is sufficient for a weak minimum, under conditions $(a),(b),(c)$.

+ See preceding footnote.

$\ddagger$ See condition (17) above. 
tion of $x, y, y^{\prime}$. On account of this theorem we will henceforth call the condition $\left.\frac{\partial^{2} f}{\partial y^{\prime 2}}\right]_{C}>0$ Legendre's sufficient condition.

We will now proceed to show that Legendre's broader condition is necessary for any kind of a minimum whatever. We have seen that Legendre's sufficient condition, $\left.\frac{\partial^{2} f}{\partial y^{\prime 2}}\right]_{C}>0$ is, together with Lagrange's condition, sufficient for a limited weak minimum. But that $I$ have a minimum is equivalent to saying that $-I$ has a maximum. Hence $\left.\frac{\partial^{2} f}{\partial{y^{\prime}}^{2}}\right]_{C}<0$ is, together with Lagrange's condition, a sufficient condition for a limited weak maximum. Given now a solution of Lagrange's equation, $C$, joining $P$ and $Q$, if $\left.\frac{\partial^{2} f}{\partial y^{\prime 2}}\right]_{C}<0$ at any point of $C$, then $\left.\frac{\partial^{2} f}{\partial y^{\prime 2}}\right]_{C}<0$ for a whole interval, since $\frac{\partial^{2} f}{\partial y^{\prime 2}}$ is a continuous function of $x, y, y^{\prime}$, and $C^{\prime}$ is a continuous curve whose tangent varies continuously. But since $\left.\frac{\partial^{2} f}{\partial y^{\prime 2}}\right]_{C}<0$ is a sufficient condition for a limited weak maximum, it follows that any limited weak variation in this interval gives us a curve for which $I$ has a less value than along $C$. It follows that Legendre's broader condition, $\left.\frac{\partial^{2} f}{\partial y^{\prime 2}}\right]_{C} \geqq 0$, is a necessary condition for a minimum of any kind whatever; since otherwise we have actually found a comparison curve $C^{\prime}$ such that $I_{C^{\prime}}<I_{C}$, and this comparison curve occurs in any possible choice of realms of curves to be considered since it is a limited weak variation of $C$, the tangents to which can be made, if necessary, to turn continuously.* On account of this theorem we shall henceforth call the condition $\left.\frac{\partial^{2} f}{\partial y^{\prime 2}}\right]_{C} \geqq 0$ Legendre's necessary condition.

It seems fitting at this point to call attention to the general usefulness of the idea of the limited variation, which has led us so easily to Legendre's necessary condition. The question

\footnotetext{
* Or even analytically, by the now well known method originally due to Schwarz.
} 
of geodetics in the surface theory, and many questions in mechanics, notably the Hamilton-Jacobi theory, require only the use of limited variation, and indeed it is peculiarly suited to the needs of these and other similar subjects. Its greater simplicity in statement and treatment commend it above methods commonly employed, and its results are more easily obtained and grasped. For instance, in the surface theory, any geodetic line actually renders the integral of length on the surface a limited strong minimum along any portion of it which joins any two points upon it.* This statement brings into a clear light the essential characteristics of a geodetic as a "shortest" line, and its application to such special surfaces as the sphere and the anchor ring are particularly vivid.

\section{Unlimited Variation. Production of Jacobi's Con- DITION FROM WeIERSTRASS'S CONDITION. $\dagger$}

Jacobi's condition was originally erroneously stated as a sufficient condition, and was shown by Weierstrass to be insufficient for strong minima. Thus far we bave obtained Weierstrass's condition (Section 1) as sufficient for a strong minimum under the necessary conditions $(a),(b),(c)$ of Section 1 . We now propose to obtain Jacobi's condition as a necessary condition for any (unlimited) mininum from consideration and elimination of these conditions $(a),(b),(c)$. We shall then show that Jacobi's condition (together with Lagrange's and Legendre's) is a sufficient condition for weak minima, the Weierstrass condition being unnecessary. Thus the Jacobi condition precisely covers the introduction of the unlimited variation, for exactly analogous results have been obtained for limited variation (Section 2) with its omission.

We will suppose the Lagrange condition satisfied, since it is a first necessary condition for any discussion. Let the supposed solution $C$ of our problem be the curve

$$
y=y(x)
$$

joining $P:\left(x_{0}, y_{0}\right)$ and $Q:\left(x_{1}, y_{1}\right)$. The Lagrange equation

$$
y^{\prime \prime} \frac{\partial^{2} f}{\partial y^{\prime 2}}+y^{\prime} \frac{\partial^{2} f}{\partial y \partial y^{\prime}}+\frac{\partial^{2} f}{\partial x \partial y^{\prime}}-\frac{\partial f}{\partial y}=0
$$

\footnotetext{
* In this particular theorem, of course, no new fact is involved.

† In connection with this section, see Osgood, Transactions Amer. Math. Soc., l. c., p. 166.
} 
has in general a two-parameter family of solutions

$$
y=\phi\left(x, c_{1}, c_{2}\right)
$$

where $\phi$ is an analytic function of $x, c_{1}, c_{2}$, if, as supposed, $f$ is analytic in $x, y, y^{\prime}$.

Let us now suppose that $c_{1}$ and $c_{2}$ are so chosen that

$$
\phi(x, 0,0) \equiv y(x)
$$

so that for $c_{1}=0, c_{2}=0$, our curve $C$ results. Further let $c_{1}=t \gamma_{1}$ and $c_{2}=t \gamma_{2}$. Then (3) becomes

$$
y=\phi\left(x, t \gamma_{1}, t \gamma_{2}\right) \text {. }
$$

Since $\phi$ is analytic in $c_{1}$ and $c_{2}$ we have, when $\gamma_{1}$ and $\gamma_{2}$ are regarded as constant,

$$
\left\{\begin{aligned}
y & =\phi\left(x, t \gamma_{1}, t \gamma_{2}\right)=\Phi(x, t), \quad \text { say } \\
& =\Phi(x, 0)+t \frac{\partial \Phi(x, 0)}{\partial t}+\frac{t^{2}}{2 !} \frac{\partial^{2} \Phi(x, 0)}{\partial t^{2}}+\cdots \\
& =y(x)+t \phi_{1}(x)+t^{2} \phi_{2}(x)+\cdots
\end{aligned}\right.
$$

which converges uniformly for sufficiently small values of $t$.

Now, regarding $\gamma_{1}$ and $\gamma_{2}$ as constant, and (6) as our oneparameter family of solutions required by condition $(b)$, Section 1 , let us set

$$
p=\frac{\partial \Phi(x, t)}{\partial x}=\frac{d y(x)}{d x}+t \frac{d \phi_{1}(x)}{d x}+t^{2} \frac{d \phi_{2}(x)}{d x}+\cdots
$$

If now we can solve (6) for $t$ as a single valued function of $y$ and $x$, we can insert the value found in (7) and have $p$ defined as a single valued function of $y$ and $x, i$. e., of the position; and this will hold for sufficiently small values of $t$, since the series used are then uniformly convergent, $i$. e., it will hold for all points in a suitably chosen neighborhood of $C$. Such a solution will then satisfy the purposes of the Weierstrass-Hilbert theory. But for this (compare condition $c$, Section I) it is only necessary that

$$
\phi_{1}(x) \neq 0 \text {. }
$$

But 
whence

$$
\left.\phi_{1}(x)=\frac{d \Phi(x, t)}{d t}\right]_{t=0}
$$

$$
\begin{aligned}
\phi_{1}(x) & =\frac{\partial \phi\left(x, c_{1}, c_{2}\right)}{\partial c_{1}} \frac{d c_{1}}{d t}+\frac{\partial \phi\left(x, c_{1}, c_{2}\right)}{\partial c_{2}} \frac{d c_{2}}{d t} \\
& =\frac{\partial \phi}{\partial c_{1}} \gamma_{1}+\frac{\partial \phi}{\partial c_{2}} \gamma_{2}=\eta_{1} \gamma_{1}+\eta_{2} \gamma_{2},
\end{aligned}
$$

where $\quad \eta_{1} \equiv \frac{\partial \phi}{\partial c_{1}}, \quad \eta_{2} \equiv \frac{\partial \phi}{\partial c_{2}}$.

If then we can so choose $\gamma_{1}$ and $\gamma_{2}$ that $\phi(x)$ does not vanish between $x_{0}$ and $x_{1}$ the Weierstrass-Hilbert theory can be applied.

And further, the possibility of such a choice is necessary for the existence of a minimum. For, consider the family of extremals out of (3) which pass through $\left(x_{0}, y_{0}\right)$ and suppose that $\gamma_{1}$ and $\gamma_{2}$ are so chosen that (6) represents this family.* If then $\phi_{1}(x)$ vanishes at any point $R$ between $x_{0}$ and $x_{1}$ then the equations

$$
\left\{\begin{array}{l}
y=\Phi(x, t), \\
\frac{d \Phi(x, t)}{d t}=0 \\
y=y(x) \equiv \Phi(x, 0)
\end{array}\right.
$$

are satisfied at this point, since

$$
\left.\frac{d \Phi(x, t,)}{d t}\right]_{t=0}=\phi_{1}(x) .
$$

Hence the envelope of the above family of extremals through $\left(x_{0}, y_{0}\right)$ cuts the curve $C$ at any such point as $R$.

If now this envelope be a single point, then compare the value $I_{C}$ with $I_{\bar{C}}$ where $\bar{C}$ is any other extremal of the family. Since $J$ is independent of the path and since $J$ reduces to $I$ for all extremals, it follows that $C$ does not render $I$ a proper (unlimited) minimum of any sort whatever, between $P$ and $R$ or

\footnotetext{
* It can be shown from the considerations at the end of this Section, that this is the best possible choice.
} 
between $P$ and any point beyond $R$. For $\left.\left.I_{C}\right]_{P}^{R}=I_{\bar{\sigma}}\right]_{P}^{R}$ and $\bar{C}$ is an analytic weak variation of $C$.

If on the other hand the envelope is a curve, then compare $\left.I_{C}\right]_{P}^{R}$ with $\left.\left.I_{C}\right]_{P}^{R^{\prime}}+I_{E}\right]_{R^{\prime}}^{R}$, where $\bar{C}$ is any other extremal of the family, $E$ the envelope, and $R^{\prime}$ the point where $\bar{C}$ meets $E$. Since the envelope is tangent at every point to an extremal of the family, it follows, as before, that $J_{E}=I_{E}$ and hence $\left.\left.\left.I_{C}\right]_{P}^{R}=I_{\bar{C}}\right]_{P}^{R^{\prime}}+I_{E}\right]_{R^{\prime}}^{R}$. Hence $C$ does not render $I$ a proper * (unlimited) minimum between $P$ and $R$ nor between $P$ and any point beyond $R$.

It follows that if we can so choose $\gamma_{1}$ and $\gamma_{2}$ that

$$
\phi_{1}(x) \neq 0, \quad x_{0}<x \leqq x_{1},
$$

the Weierstrass-Hilbert theory can be applied; and further the possibility of such a choice is a necessary condition for the existence of an (unlimited) minimum of any sort.

We know that

$$
y=\phi\left(x, c_{1}, c_{2}\right)=\Phi(x, t)
$$

must satisfy Lagrange's equation

$$
L(y) \equiv \frac{d}{d x}\left(\frac{\partial f}{\partial y^{\prime}}\right)-\frac{\partial f}{\partial y}=0
$$

(13) $0=L(\Phi(x, t))=L\left(y(x)+t \phi_{1}(x)+t^{2} \phi_{2}(x)+\cdots\right)$

$\left.\left.=L(y(x))+\frac{d L\left(y(x)+t \phi_{1}(x)+\cdots\right)}{d t}\right]_{t=0} t+\frac{d^{2} L}{d t^{2}}\right]_{t=0} \frac{t^{2}}{2 !}+\cdots$

for all sufficiently small values of $t$. Hence the coefficient of $t$ must vanish separately,

$$
\left.\frac{d}{d t}\left\{L\left(y(x)+t \phi_{1}(x)+t^{2} \phi_{2}(x)+\cdots\right)\right\}\right]_{t=0}=0 .
$$

But $L(\xi)$ involves $\xi, \frac{d \xi}{d x}, \frac{d^{2} \xi}{d x^{2}}$. Hence we have

\footnotetext{
* It can indeed be shown that $C$ does not even render $I$ an improper minimum, for the envelope cannot be an extremal. It is to be noticed that all the above work holds even when the extremals out the envelope.
} 


$$
\left.\frac{\partial L(\xi)}{\partial \frac{d^{2} \xi}{d x^{2}}} \frac{d \frac{d^{2} \xi}{d x^{2}}}{d t}+\frac{\partial L(\xi)}{\partial \frac{d \xi}{d x}} \frac{d \frac{d \xi}{d x}}{d t}+\frac{\partial L(\xi)}{\partial \xi} \frac{d \xi}{d t}\right]_{t=0}=0
$$

where

$$
\xi \equiv y(x)+t \phi_{1}(x)+t^{2} \phi_{2}(x)+\cdots ;
$$

or

$$
\frac{\partial L(y)}{\partial y^{\prime \prime}} \phi_{1}^{\prime \prime}(x)+\frac{\partial L(y)}{\partial y^{\prime}} \phi_{1}^{\prime}(x)+\frac{\partial L(y)}{\partial y} \phi_{1}(x)=0
$$

an equation which must be satisfied by $y=y(x)$. Using the definition of $L(y)$, we find by a simple calculation that $\phi_{1}(x)$, and hence both $\eta_{1}$ and $\eta_{2}$, must satisfy the following linear differential equation of the second order :

$$
f_{y^{\prime} y^{\prime}} \cdot \eta^{\prime \prime}+\frac{d f_{y^{\prime} y^{\prime}}}{d x} \eta^{\prime}+\left(\frac{d f_{y y^{\prime}}}{d x}-f_{y y}\right) \eta=0
$$

where

$$
\eta^{\prime \prime} \equiv \frac{d^{2} \eta}{d x^{2}}, \quad f_{y^{\prime} y^{\prime}} \equiv \frac{\partial^{2} f}{\partial y^{\prime 2}}, \quad \text { etc. }
$$

as usual. But (17) is precisely Jacobi's equation; and Jacobi's whole condition consisted in finding whether an integral of this equation

$$
\phi_{1}(x)=\gamma_{1} \eta_{1}+\gamma_{2} \eta_{2}
$$

existed, which did not vanish between $x_{0}$ and $x_{1}$. Hence the above are precisely Jacobi's conditions, except that the equality sign in our condition must be omitted in the usual discussion, and the corresponding case made a subject of further investigation.

The rest of the results announced at the beginning of the article now follow readily, and will be left to the reader.

\section{Summary of Conditions.}

Collecting our results, we may say that the following conditions hold : 


\begin{tabular}{|c|c|c|c|c|}
\hline & \multicolumn{2}{|c|}{ Limited Variations. } & \multicolumn{2}{|c|}{ Unlimited Variations. } \\
\hline & Weak. & Strong. & Weak. & Strong. \\
\hline Necessary. & $\begin{array}{c}\text { Lagrange's, } \\
\text { Legendre's } \\
\text { necessary. }\end{array}$ & $\begin{array}{c}\text { Lagıange's, } \\
\text { Legendre's } \\
\text { necessary, } \\
\text { Weierstrass's } \\
\text { necessary. }\end{array}$ & $\begin{array}{l}\text { Lagrange's, } \\
\text { Legendre's } \\
\text { necessary, } \\
\text { Jacobi's. }\end{array}$ & $\begin{array}{l}\text { Lagrange's, } \\
\text { Legendre's } \\
\text { necessary, } \\
\text { Jacobi's, } \\
\text { Weierstrass's } \\
\text { necessary. }\end{array}$ \\
\hline Sufficient. & $\begin{array}{l}\text { Lagrange's, } \\
\text { Legendre's } \\
\text { sufficient. }\end{array}$ & $\begin{array}{l}\text { Lagrange's, } \\
\text { Legendre's } \\
\text { sufficient, } \\
\text { Weierstrass's } \\
\text { sufficient. }\end{array}$ & $\begin{array}{l}\text { Lagrange's, } \\
\text { Legendre's } \\
\text { sufficient, } \\
\text { Jacobi's. }\end{array}$ & $\begin{array}{l}\text { Lagrange's, } \\
\text { Legendre's } \\
\text { sufficient, } \\
\text { Jacobi's, } \\
\text { Weierstrass's } \\
\text { sufficient. }\end{array}$ \\
\hline
\end{tabular}

It is seen on glancing at the table that from the simple conditions (Lagrange's and Legendre's) for limited weak variation we proceed to any other case by adding Weierstrass's conditions in the case of a strong minimum, and Jacobi's in case of an unlimited minimum, only. It is to be hoped that advances may be made in bringing the necessary and sufficient conditions more closely together or into entire coincidence. The above table represents substantially the present known conditions, in the belief of the writer.

In special problems the irksomeness of these conditions can sometimes be circumvented. For instance, given a problem in which $\frac{\partial^{2} f}{\partial y^{\prime 2}}>0$ for all values of $x, y, y^{\prime}$, then the necessary and sufficient condition for a limited strong minimum is the possibility of finding a solution of Lagrange's equation joining the two given end points. Such is the case in the geodetic problem and also in the integral which leads to Hamilton's principle; and in each of these cases, fortunately, a limited strong minimum is all that is desired. Similar simplification occurs in every case when $\frac{\partial^{2} f}{\partial y^{\prime 2}}>0$ for all $x, y, y^{\prime}$. For then Legendre's and Weierstrass's conditions are always satisfied, and may be abstracted from the above table. For this reason Hilbert has called a problem in which $\frac{\partial^{2} f}{\partial y^{\prime 2}}>0$ for all $x, y, y^{\prime}$ contained in a singly connected region $R$, in which the given end points lie, a "regular" problem of the calculus of variations. 


\section{Conclusion. Hilbert's Existence Theorem.}

A misunderstanding has sometimes arisen in regard to Hilbert's existence proof. Its purpose is not to establish further conditions nor to solve more general problems, but is to reassure us as to the possibility of satisfying the conditions already found, in the more favorable cases. We have seen that a necessary condition for any minimum was the possibility of finding a solution of Lagrange's equation joining the two end points $P$ and $Q$; and that this is a sufficient condition for a limited strong minimum, in a regular problem. That such a solution exists, even in the most favorable cases, is by no means certain from the ordinary theory of differential equations. It was the original purpose of Hilbert's proof (merely) to show that, in this most favorable case of a regular problem, a curve must exist which renders our integral an unlimited strong minimum, compared with all continuous comparison curves ; and that the minimizing curve is composed of a finite number of pieces of extremals. Hilbert's existence theorem may therefore properly be called a theorem in differential equations.

At present the results cannot be said to demonstrate more than that a curve exists which renders the integral an improper minimum. This result cannot be extended in general, and it remains to show that Lagrange's equation is necessary, not only for proper, but also for improper minima.

In conclusion, the author desires to enter protest against the extreme complication recently introduced in some quarters into the essentially simple subject of the calculus of variations. In the case of the only modern text-book on the theory,* this condition is so exaggerated as to essentially mar the usefulness of the book, in that many who would otherwise interest themselves in the subject, are repelled by the style and treatment. It is to be sincerely regretted, in the opinion of the writer, that this tendency has been followed in some of the recent memoirs.

YALE UNIVERSITY, June, 1902.

* Kneser, Variationsrechnung. 